\title{
Frequency and Types of Ciliary Ultrastructural Defects in Patients With Suspected Primary Ciliary Dyskinesia Symptoms Analyzed by Transmission Electron Microscopy
}

\section{Mitra Rezaei}

Virology Research center, National Research Institute of Tuberculosis and Lung Disease (NRITLD), Shahid Beheshti University of Medical Sciences, Tehran, Iran

\section{Amirali Soheili}

Medical student research committee, School of Medicine, Shahid Beheshti University of Medical

Sciences, Tehran, Iran

\section{Atefeh Fakharian}

Chronic Respiratory Diseases Research Center (CRDRC), National Research Institute of Tuberculosis and Lung Diseases (NRITLD), Shahid Beheshti University of Medical Sciences, Tehran, Iran

\section{Hamid Jamaati}

Department of Internal Medicine, National Research Institute of Tuberculosis and Lung Disease (NRITLD), Masih Daneshvari Hospital, Shahid Beheshti University of Medical Sciences

\section{Jahangir Ghorbani}

Chronic Respiratory Diseases Research Center (CRDRC), National Research Institute of Tuberculosis and Lung Diseases (NRITLD), Shahid Beheshti University of Medical Sciences, Tehran, Iran

\section{Hossein Toreyhi}

Medical student research committee, School of Medicine, Shahid Beheshti University of Medical Sciences, Tehran, Iran

\section{Seyed Ali Ziai ( $\nabla$ saziai@gmail.com )}

Department of Pharmacology, School of Medicine, Shahid Beheshti University of Medical Sciences, Tehran, Iran

\section{Research Article}

Keywords: PCD, SCD, TEM, primary ciliary dyskinesia, secondary ciliary dyskinesia, dynein arm, IDA, ODA, transmission electron microscopy, cilium, cilia, ciliary ultrastructure

Posted Date: January 18th, 2021

DOI: https://doi.org/10.21203/rs.3.rs-141285/v1 
License: (c) (i) This work is licensed under a Creative Commons Attribution 4.0 International License. Read Full License 


\section{Abstract}

Background: Primary ciliary dyskinesia (PCD) is a rare autosomal recessive condition of often chronic respiratory infections in early life. A useful tool for early diagnosis of such ciliary abnormalities is transmission electron microscopy (TEM). This study aimed to use TEM to examine these defects and speculate on a diagnosis.

Methods: From 2017 to 2019, all referral patients with suspected PCD symptoms were included in this study. Nasal samples were taken after exclusion of further potential differential diagnosis and prepared for TEM. The final diagnosis was based on the International Consensus Guideline for reporting transmission electron microscopy results in the diagnosis of PCD. A descriptive analysis of demographic and ciliary ultrastructural data was performed by SPSS ver 21 .

Results: Study population consisted of 37 women and 30 men (mean age $=20.34 \pm 10.7$ years). The clinical presentations were as follows: bronchiectasis: 26 patients (38.8\%); sinusitis: $23(34.3 \%)$; recurrent respiratory infection: 21 patients (31.3\%); auditory symptoms: 5 patients $(7.5 \%)$; situs inversus: 3 patients (4.4\%); productive cough: 2 patients (3\%); infertility: 2 patients (3\%); polyposis: 1 patient $(1.5 \%)$. According to TEM analysis, 12 (17\%) of patients were PCD, 11 (15.7\%) were indicating PCD cases, 26 (37.1\%) of them had no criteria of PCD and $18(25.7 \%)$ of cases had normal ciliary ultrastructure. Compound cilia and extra-tubule were reported in 29 (41.4\%) and 31(44.3\%) of patients, respectively. The outer dynein arm defect was seen in 11(16.4\%) cases and the inner dynein arm (IDA) defect was seen in $20(29.8 \%)$ cases. Two patients (3\%) had microtubular disorganization.

Conclusion: Bronchiectasis and sinusitis were the most common complications. The compound cilia and extra-tubule were the most prevalent TEM finding among all participants. However, the most prevalent hallmark diagnostic defects among PCD patients were ODA and IDA defects among PCD patients. Other diagnostic PCD tests should also be performed in patients in the indicating PCD group, those without PCD criteria, and normal patients with a highly suggestive history. Cell-culture, as well, should confirm IDA defects. This study highlights the fundamental need to consider ciliary defect among probable diagnoses and use TEM as a practical diagnostic tool.

\section{Introduction}

Chronic or recurrent respiratory tract infection is a common disease among children which can be result of compromised muco-ciliary clearance (MCC). This pathology may occur due to the thickening of mucus; most commonly seen in Cystic Fibrosis (CF) (1), impaired ciliary motility, and interaction between ciliary and respiratory mucus (2). A normal ciliary ultrastructure was firstly introduced by Afzelius consisting of a central pair of microtubules surrounded by 9 peripheral double microtubules (known as $9+2$ ) bonded together by several supporting radial spokes. Moreover, each doublet possesses outer and inner dynein arms enabling microtubules to bend the cilium (3). 
Therefore, the ultrastructural ciliary impairments include the defect of the outer or inner dynein arms, the disorder of peripheral microtubules, and the rare absence of one or both of the central pair (4). These defects could be a hereditary defect called primary ciliary dyskinesia (PCD) or a post-infectious defect called secondary ciliary dyskinesia $(S C D)(5,6)$. Diagnosis of ultrastructural ciliary defect plays a key role in both clinical decision-making and prevention of harmful results (7).

PCD is an inherited autosomal recessive disorder which present with chronic upper and lower respiratory infections starting often in the early years of life, followed by inevitable adult bronchiectasis. (8) PCD is a result of impaired ciliary beating mostly caused by an abnormal ciliary ultrastructure. These cilia also play a pivotal role during the embryonic period by determining the laterality of organs; therefore, situs inversus is a common finding among these patients. PCD may also manifest through otitis media and hearing impairment, recurrent sinusitis and male infertility, as cilia are also present in other organs $(5,8)$. The prevalence of PCD is estimated to be 1 in $10000-20000$ newborns, and is also more prevalent among consanguineous married family clusters $(5,8)$. In addition to these limited epidemiological data, phenotypic heterogeneity, unfamiliar clinical course for most general practitioners (GPs) and a lack of accurate and sensitive diagnostic methods have contributed to delayed and even incorrect diagnosis of PCD, with the result that many of these patients have been visited by GPs more than 50 times without having a definite diagnosis of PCD (5). In this cross-sectional study, we aim to assess ciliary ultrastructural defects in terms of demographic and clinical characteristics by using quantitative methods on transmission electron microscopy (TEM) to assess the findings of 87 suspected patients.

\section{Results}


Table-1: Demographic data, clinical presentation and ultrastructural ciliary findings of study groups

$\begin{array}{lllll}\text { PCD } & \text { Indicate } & \begin{array}{l}\text { No } \\ \text { criteria }\end{array} & \begin{array}{l}\text { Normal } \\ (n=12)\end{array} & \begin{array}{l}\text { Total } \\ (n=18)\end{array} \\ & (n=11) & (n=26) & & \end{array}$

Demographic data

Mean age

Gender

- $\quad$ male
- $\quad$ female
Clinical presentations

Productive cough

Bronchiectasis

Sinusitis

Infertility

Polyposis

Recurrent respiratory infection

Auditory symptoms

Situs inversus

Ultrastructural ciliary findings

\section{ODA}

- ODA absence $<25 \%$

- ODA absence from 25-50\%

- $\quad$ ODA defect (absence $>50 \%$ )

\section{IDA}

- IDA absence $<25 \%$

- IDA absence from 25-50\%

- $\quad$ IDA defect (absence >50\%)

\section{ODA \& IDA}

ODA\&IDA absence <25\%

ODA\&IDA absence from 25-
$18.6 \pm 8.8$

$23.5 \pm 12.3$

$20.9 \pm 11.1$

$18.7 \pm 10.8$

$20.3 \pm 10.7$

$6(50 \%)$

$6(50 \%)$

$2(18 \%)$

$14(54 \%)$

$8(44 \%)$

$30(45 \%)$

12(46\%) 10(56\%)

$37(55 \%)$
0

$5(42 \%) \quad 5(45 \%)$

$3(25 \%) \quad 5(45 \%)$

2(17\%)

0

6(50\%)

2(17\%)

$3(25 \%)$

0

0

$4(36 \%)$

2(18\%)

0

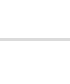

$+$

\begin{tabular}{lll}
$1(4 \%)$ & $1(5 \%)$ & $2(3 \%)$ \\
$7(27 \%)$ & $9(50 \%)$ & $26(39 \%)$ \\
\hline $10(38 \%)$ & $5(28 \%)$ & $23(34 \%)$ \\
0 & 0 & $2(3 \%)$ \\
$1(4 \%)$ & 0 & $1(1 \%)$ \\
$6(23 \%)$ & $5(28 \%)$ & $21(31 \%)$ \\
$1(4 \%)$ & 0 & $5(7 \%)$ \\
0 & 0 & $3(4 \%)$
\end{tabular}




\begin{tabular}{|c|c|c|c|c|c|}
\hline $\begin{array}{l}-\quad \text { ODA+IDA defect (absence } \\
>50 \%)\end{array}$ & $11(92 \%)$ & 0 & 0 & 0 & $11(16 \%)$ \\
\hline $\begin{array}{l}\text { Disruption of } 9+2 \text { symmetry } \\
(\text { microtubular disorganization } \\
(<=25 \%))\end{array}$ & $3(25 \%)$ & $6(54 \%)$ & $14(54 \%)$ & 0 & $23(34 \%)$ \\
\hline $\begin{array}{l}\text { Microtubular disorganization } \\
(>25 \%)\end{array}$ & $1(8 \%)$ & 0 & $1(4 \%)$ & 0 & $2(3 \%)$ \\
\hline $\begin{array}{l}\text { One of the central pair missing } \\
(<=20 \%)\end{array}$ & $2(17 \%)$ & 0 & $2(8 \%)$ & 0 & $4(6 \%)$ \\
\hline $\begin{array}{l}\text { One of the central pair missing } \\
(>20 \%)\end{array}$ & 0 & 0 & 0 & 0 & 0 \\
\hline $\begin{array}{l}\text { Both of the central pair } \\
\text { missing }(<=20 \%)\end{array}$ & $3(25 \%)$ & $3(27 \%)$ & $9(35 \%)$ & 0 & $15(22 \%)$ \\
\hline $\begin{array}{l}\text { Both of the central pair } \\
\text { missing }(>20 \%)\end{array}$ & 0 & 0 & 0 & 0 & 0 \\
\hline
\end{tabular}

Initially, 87 participants enrolled in the study, 11 samples had metaplasia of squamous cells, 8 samples were poorly preserved, and one sample did not have adequate cilia to examine.(Figure 1) Therefore, 20 patients were excluded and 67 participants consisted of the final study population. Among these patients, $30(42.9 \%)$ were male and $37(52.9 \%)$ were female. They are between $4-47$ years of age (mean age $=$ $20.34 \pm 10.7$ years). Demographic characteristics and ciliary ultrastructural defects of the cases are shown in Table-3. The TEM analysis revealed that this population consists of 12(17\%) PCD cases, $11(15.7 \%)$ indicate PCD case, $26(37.1 \%)$ patients had no criteria of PCD and $18(25.7 \%)$ were normal cases. Moreover, other ciliary defects were also seen: $31(44.3 \%)$ had extra-tubule and $29(41.4 \%)$ had compound cilia.

Productive cough was only reported in 2 patients (3\%). Recurrent respiratory infection was seen in 21 patients (31.3\%). Also, 26(38.8\%) and 23(34.3\%) cases had bronchiectasis and sinusitis, respectively. Infertility was reported in two cases and polyposis was reported in only one case. (Table-1)

Situs inversus was only seen in PCD cases (3 patients) and auditory symptoms were reported mostly among PCD and indicate PCD group (4 out of all 5 cases presenting with these symptoms). (Table-2) 


\begin{tabular}{|c|c|c|c|c|c|}
\hline $\begin{array}{l}\text { Case } \\
\text { No. }\end{array}$ & Age & Gender & Clinical presentations & TEM ultrastructure & Diagnosis \\
\hline 29 & 14 & Male & $\begin{array}{l}\text { Recurrent respiratory } \\
\text { infection }\end{array}$ & $\begin{array}{l}\text { ODA+IDA defect/ extra tubule + } \\
\text { compound tubule }\end{array}$ & PCD \\
\hline 49 & 17 & Female & $\begin{array}{l}\text { Recurrent respiratory } \\
\text { infection }\end{array}$ & ODA+IDA defect/ extra tubule & PCD \\
\hline 70 & 10 & Female & $\begin{array}{l}\text { Recurrent respiratory } \\
\text { infection+ Auditory } \\
\text { symptoms }\end{array}$ & $\begin{array}{l}\text { ODA+IDA defect/ extra tubule + } \\
\text { compound tubule }\end{array}$ & PCD \\
\hline 32 & 13 & Male & $\begin{array}{l}\text { Recurrent respiratory } \\
\text { infection }\end{array}$ & ODA+IDA defect & PCD \\
\hline 33 & 19 & Male & Sinusitis+ Situs inversus & ODA+IDA defect & PCD \\
\hline 50 & 4 & Female & $\begin{array}{l}\text { Recurrent respiratory } \\
\text { infection }\end{array}$ & ODA+IDA defect & PCD \\
\hline 58 & 24 & Female & $\begin{array}{l}\text { Bronchiectasis+ } \\
\text { Sinusitis+ Infertility }\end{array}$ & ODA+IDA defect/ extra tubule & PCD \\
\hline 65 & 18 & Female & Bronchiectasis & ODA+IDA defect & PCD \\
\hline 46 & 32 & Male & $\begin{array}{l}\text { Bronchiectasis+ } \\
\text { Sinusitis+ Infertility+ } \\
\text { Situs inversus }\end{array}$ & ODA+IDA defect/ extra tubule & PCD \\
\hline 82 & 24 & Female & Bronchiectasis & $\begin{array}{l}\text { ODA+IDA defect/ compound } \\
\text { tubule }\end{array}$ & PCD \\
\hline \multirow[t]{2}{*}{86} & 34 & Male & $\begin{array}{l}\text { Recurrent respiratory } \\
\text { infection+ Situs inversus }\end{array}$ & $\begin{array}{l}\text { IDA defect+ microtubular } \\
\text { disorganization/ }\end{array}$ & PCD \\
\hline & & & & $\begin{array}{l}\text { both of the central pair } \\
\text { missing+ extra tubule }+ \\
\text { compound tubule }\end{array}$ & \\
\hline 87 & 15 & Male & $\begin{array}{l}\text { Bronchiectasis+ Auditory } \\
\text { symptoms }\end{array}$ & $\begin{array}{l}\text { ODA+IDA defect/ compound } \\
\text { tubule }\end{array}$ & PCD \\
\hline
\end{tabular}

\section{Discussion}

This was the first study conducted in Iran to evaluate ciliary ultrastructural defects among patients presenting with symptoms attributable to PCD. Early diagnosis of PCD is of great importance as 
appropriate clinical intervention and early treatment prevent permanent bronchiectasis and other insidious lung tissue damage (9).

According to the European Respiratory Society Guidelines for the diagnosis of primary ciliary dyskinesia, it is highly recommended that patients with recurrent wet cough, chronic rhinitis, persistent middle ear disease, congenital heart disease, neonatal respiratory infections in infants and a history of intensive neonatal treatment be screened for PCD. In addition, PCD patients' siblings should also undergo PCD investigations $(10,11)$. The most prominent symptoms among patients were bronchiectasis $(39 \%)$ and persistent sinusitis (34\%). However, in the PCD group, persistent respiratory infection was even greater than those symptoms reported (50\%).

PCD and SCD may have similar symptoms, but TEM findings are permanent and seen in most of the cilia in PCD patients. Secondary changes, however, could be the result of former infections and would recover by the time or culture of the sample $(6,12,13)$. Compound cilia $(41.4 \%)$ and extra-tubules $(44.3 \%)$ are examples of SCD identified in almost half of the sample population (Figure.2).

Furthermore, some changes, such as microtubular disorganization and central pair abnormalities, may be common between PCD and SCD. Therefore, to confirm this diagnosis, they should present with other specific PCD criteria. Repeating the biopsy and considering a sufficient healthy interval before sampling also help to differentiate between PCD and SCD more precisely (14). IDA defect is also among the overlapping appearance seen in almost $30 \%$ of our samples, probably due to the difficulty of seeing IDA by TEM (15-17). (Table-3) (Figure.3).

It is therefore highly suggested that the biopsy be iterated specifically for this group (18). In addition, in the event of an isolated IDA defect, the cultivation of the sample may result in a reversal and therefore exclude the diagnosis of PCD (6). Likewise, considerable attention must be paid to the ultrastructure at the tip and base of the cilia to prevent misinterpretation as ultrastructural defect (14).(Figure 4).

To date, PCD diagnosis remains a significant medical challenge in resource-limited regions. Although TEM was previously thought to be a gold standard test, there is currently no stand-alone PCD test and there are also a variety of diagnostic approaches in different countries based on their local guidelines (19).

Several techniques are required to confirm PCD diagnosis, as well. Saccharine testing was previously done for PCD screening (20), although this is not reliable anymore (20). To date, nasal nitric oxide (nNO) and high-speed video microscopy analysis (HSVA) have been used in combination with TEM to confirm a PCD diagnosis based on local diagnostic algorithms. However, the European Respiratory Society Guideline (10) labels nNO (for patients under the age of 6 years) and HSVA as a "weak recommendation" and still strongly advocates TEM. Due to the lack of a local guideline in our region, we recommend that TEM be a reliable tool because of its high specificity. However, up to 30 percent of PCD patients have normal TEM results (14). Furthermore, by using TEM alone, there is no confirmed diagnosis for patients with" Class 2 "defects (indicate PCD). We therefore suggest that other tests should be performed for these 
patients, along with TEM repeat or sample cultivation, to make further decisions (14). Further studies are needed to establish a local guideline in western Asian countries.

These patients would have a normal joyful life only if they are diagnosed on-time and given proper antibiotic treatment in each respiratory infections. Regular physiotherapy is useful, as well (21) On the other hand, recurrent infections will result in respiratory complications, including recurrent pneumonitis, bronchiectasis, parenchymal fibrosis, hypoxemia induced by ventilation / perfusion mismatch and right ventricular failure, if they remain undiagnosed. Hence, early diagnosis would prevent irreversible consequent complications caused during clinical course and also reduce the burden of disease and its great cost for the health system (22).

Our work has led us to conclude that among suspected PCD patients the most common ultrastructural ciliary defects were extra-tubule and compound cilia. However, the most prevalent hallmark diagnostic defects were ODA and IDA defect among PCD patients. Further studies are needed to establish a local guideline in western Asian countries.

\section{Methods}

\section{Study design and patient enrollment:}

This prospective cohort study was conducted in referral patients from several centers to TEM of central laboratory, School of Medicine, Shahid Beheshti University of Medical Sciences from 2017 to 2019. These patients had at least one of the suspected symptoms of PCD listed in Table-4 or a positive history of PCD in the family (particularly in consanguineous marriages and siblings). 


\section{Table-4: Suspected presentations of PCD}

1. Respiratory distress in newborn

2. Situs inversus, heterotaxy, poly-or asplenia

3. Chronic otitis media

4. hearing loss

5. neonatal pneumonia

6. Chronic nasal congestion and mucopurulent rhinitis

7. Nasal polyposis

8. Chronic pan-sinusitis

9. Chronic wet cough

10. Recurrent pneumonia

11. bronchiectasis of unknown etiology

12. Airway obstruction, gas trapping or treatment-resistant asthma

13. Unexplained male and female infertility

14. Hydrocephalus

15. Retinitis pigmentosa

Patients were subsequently tested for probable differential diagnoses, including asthma (using both salbutamol and methacholine challenge tests), cystic fibrosis, immunological disorders and chronic gastroesophageal reflux (depending on their past medical history) and excluded from the study group.

Overall, 87 patients were enrolled in this study and tested for ciliary ultrastructural defects. Informed consents were taken from the participants. The current study has also been approved by the Ethics Committee of medical school, Shahid Beheshti University of medical science

(Code=IR.SBMU.MSP.REC.1397.382). All methods were carried out in accordance with relevant guidelines and regulations.

A questionnaire designed by the expert medical team was filled out by all participants. This questionnaire provided demographic data (age and sex) and their clinical presentations including chronic productive cough, recurrent sinusitis, recurrent respiratory infections, auditory symptoms, situs inversus and infertility in both genders.

\section{TEM analysis:}


Ciliary respiratory mucosa was achieved by an expert Ear, Nose, Throat (ENT) specialist. It is important to note that sampling was avoided in patients who currently have upper or lower respiratory tract infections and were asked to return without any respiratory symptoms after 6 weeks. Nasal samples were fixed by glutaraldehyde $2.5 \%$ and then second fixation was performed using osmium tetroxide $1 \%$ in $0.1 \mathrm{M}$ veronal acetate buffer. The samples were dehydrated by passing through the ethanol series and then embedded in the epoxy-containing resin. The ultrathin sections of lead citrate and uranyl acetate have been stained to provide an appropriate ultrastructural view. These sections were evaluated using a ZEISS EM900 TEM to detect ciliary ultrastructural defects. For each case, at least 50 intact cilia from healthiest areas were examined.

In addition, the non-detectable cilia sample in the light-microscopic assessment such as squamous cell metaplasia, samples with an insufficient number of cilia (less than 50 sections for each patient) and poorly preserved samples that are barely useful in ciliary ultrastructural assessment were also excluded.

(Figure 1)

Ultrastructural defects are classified into two main classes, according to the International Consensus Guideline for reporting transmission electron microscopy results in the diagnosis of PCD (14). (Table-2)

\section{Class 1 defects include:}

1. Outer dynein arm (ODA) defect: absence of more than 7 out of 9 arms in each cilium which must be seen in more than $50 \%$ of cilia.

2. Both outer (ODA) and inner (IDA) dynein arms defect: ODA defect with IDA defect defined as absence of more than 5 out of 9 arms in each cilium which must be seen in more than $50 \%$ of cilia.

3. Microtubular disorganization and IDA defect: Microtubular disorganization is defined as the disruption $9+2$ symmetry in more than $25 \%$ of cilia.

\section{Class 2 defects include:}

1. Central complex defect: is defined as absence of one or two of the central microtubules in more than $20 \%$ of examined cilia or lateral transposition of central pair. Also abnormal count like $8+1$ are in this category.(Figure.5)

2. Mislocalisation of basal bodies with few or no cilia: very low count of cilia along with basal bodies failure to dock the apical surface of each cilium.

3. Microtubular disorganization with IDA present: in this criteria disruption $9+2$ symmetry is seen in less than $25 \%$ of cilia.

4. ODA absence from $25-50 \%$ of cross sections

5. Combined IDA and ODA absence from $25-50 \%$ of cross sections

Therefore, based on these findings, patients were classified into 4 groups (Figure 1) 
1. PCD: existence of at least one of the class 1 defects.

2. Indicate PCD: existence of at least one of the class 2 defects with other supporting evidence.

3. No criteria (of PCD): having other ciliary ultrastructural defects which are not classified in class 1 or 2.

4. Normal: normal ciliary ultrastructure.

\section{Statistical analysis:}

Data were recorded in Microsoft Excell 2019 and then imported to the SPSS ver.21. Data analysis was conducted using descriptive analysis of demographic and ciliary ultrastructural characteristics of each patients after splitting them in to PCD, indicate PCD, No criteria and Normal groups.

\section{Declarations}

\section{Author contributions statement}

M Rezaei, A Fakharian, and H.R Jamaati conceived of the presented idea. S.A Ziai supervised the project. $J$ Ghorbani was involved in planning and provided critical feedback and helped shape the research. $M$ Rezaei and A Soheili designed the study and along with $\mathrm{H}$ Toreyhi contributed to the final version of the manuscript. All authors discussed the results and commented on the final manuscript.

\section{Data availability}

The data supporting the findings of this study are available on request from the corresponding author. However, they are not publicly available due to containing information that might compromise the privacy of study group.

\section{Competing interests:}

The authors declare no competing interests

\section{Reference}

1. Bergeron C, Cantin AM, editors. Cystic fibrosis: Pathophysiology of lung disease. Seminars in respiratory and critical care medicine; 2019: Thieme Medical Publishers.

2. de longh RU, Rutland J. Ciliary defects in healthy subjects, bronchiectasis, and primary ciliary dyskinesia. American journal of respiratory and critical care medicine. 1995;151(5):1559-67.

3. Afzelius B. Cilia-related diseases. The Journal of Pathology: A Journal of the Pathological Society of Great Britain and Ireland. 2004;204(4):470-7.

4. Ehouman A, Pinchon M, Escudier E, Bernaudin J. Ultrastructural abnormalities of respiratory cilia. Virchows Archiv B. 1985;48(1):87. 
5. Abitbul R, Amirav I, Blau H, Alkrinawi S, Aviram M, Shoseyov D, et al. Primary ciliary dyskinesia in Israel: Prevalence, clinical features, current diagnosis and management practices. Respiratory medicine. 2016;119:41-7.

6. Dixon M, Shoemark A. Secondary defects detected by transmission electron microscopy in primary ciliary dyskinesia diagnostics. Ultrastructural pathology. 2017;41(6):390-8.

7. Rubbo B, Lucas JS. Clinical care for primary ciliary dyskinesia: current challenges and future directions. European Respiratory Review. 2017;26(145).

8. Lucas JS, Burgess A, Mitchison HM, Moya E, Williamson M, Hogg C. Diagnosis and management of primary ciliary dyskinesia. Archives of disease in childhood. 2014;99(9):850-6.

9. Karakoc GB, Yilmaz M, Altintas DU, Kendirli SG. Bronchiectasis: still a problem. Pediatric pulmonology. 2001;32(2):175-8.

10. Lucas JS, Barbato A, Collins SA, Goutaki M, Behan L, Caudri D, et al. European Respiratory Society guidelines for the diagnosis of primary ciliary dyskinesia. European Respiratory Journal. 2017;49(1).

11. Pizzi S, Cazzato S, Bernardi F, Mantovani W, Cenacchi G. Clinico-pathological evaluation of ciliary dyskinesia: diagnostic role of electron microscopy. Ultrastructural pathology. 2003;27(4):243-52.

12. Mygind N, Pedersen M, Nielsen M. Primary and secundary ciliary dyskinesia. Acta oto-laryngologica. 1983;95(5-6):688-94.

13. Al-Rawi MM, Edelstein DR, Erlandson RA. Changes in nasal epithelium in patients with severe chronic sinusitis: a clinicopathologic and electron microscopic study. The Laryngoscope. 1998;108(12):181623.

14. Shoemark A, Boon M, Brochhausen C, Bukowy-Bieryllo Z, De Santi MM, Goggin P, et al. International consensus guideline for reporting transmission electron microscopy results in the diagnosis of primary Ciliary dyskinesia (BEAT PCD TEM criteria). European Respiratory Journal. 2020;55(4).

15. Escudier E, Couprie M, Duriez B, Roudot-Thoraval F, Millepied M-C, Prulière-Escabasse V, et al. Computer-assisted analysis helps detect inner dynein arm abnormalities. American journal of respiratory and critical care medicine. 2002;166(9):1257-62.

16. Keith Funkhouser III W, Niethammer M, Carson JL, Burns KA, Knowles MR, Leigh MW, et al. A new tool improves diagnostic test performance for transmission em evaluation of axonemal dynein arms. Ultrastructural pathology. 2014;38(4):248-55.

17. Papon J-F, Bassinet L, Cariou-Patron G, Zerah-Lancner F, Vojtek A-M, Blanchon S, et al. Quantitative analysis of ciliary beating in primary ciliary dyskinesia: a pilot study. Orphanet journal of rare diseases. 2012;7(1):78.

18. Smith CM, Hirst RA, Bankart MJ, Jones DW, Easton AJ, Andrew PW, et al. Cooling of cilia allows functional analysis of the beat pattern for diagnostic testing. Chest. 2011;140(1):186-90.

19. Rumman N, Jackson C, Collins S, Goggin P, Coles J, Lucas JS. Diagnosis of primary ciliary dyskinesia: potential options for resource-limited countries. European Respiratory Review. 2017;26(143). 
20. Barbato A, Frischer T, Kuehni C, Snijders D, Azevedo I, Baktai G, et al. Primary ciliary dyskinesia: a consensus statement on diagnostic and treatment approaches in children. European Respiratory Journal. 2009;34(6):1264-76.

21. Bush A, Cole P, Hariri M, Mackay I, Phillips G, O'callaghan C, et al. Primary ciliary dyskinesia: diagnosis and standards of care. European Respiratory Journal. 1998;12(4):982-8.

22. Rossman CM, Newhouse MT. Primary ciliary dyskinesia: evaluation and management. Pediatric pulmonology. 1988;5(1):36-50.

\section{Tables}

Due to technical limitations, table 3 is only available as a download in the Supplemental Files section.

\section{Figures}

\section{7 patients with suspected symptoms of PCD}

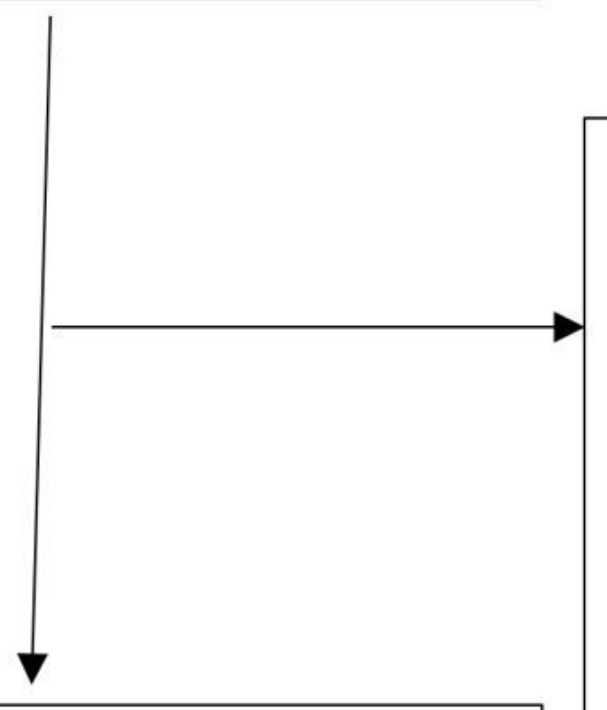

Final study population: 67(30Male, 37Female)

1. PCD: 12

2. Indicate PCD: 11

3. No criteria: 26

4. Normal: 18

\section{Exclude:}

1. Squamous cell metaplasia: 11

2. Poorly preserved samples: 8

3. Inadequate ciliary count: 1

\section{Figure 1}


Inclusion criteria and classification

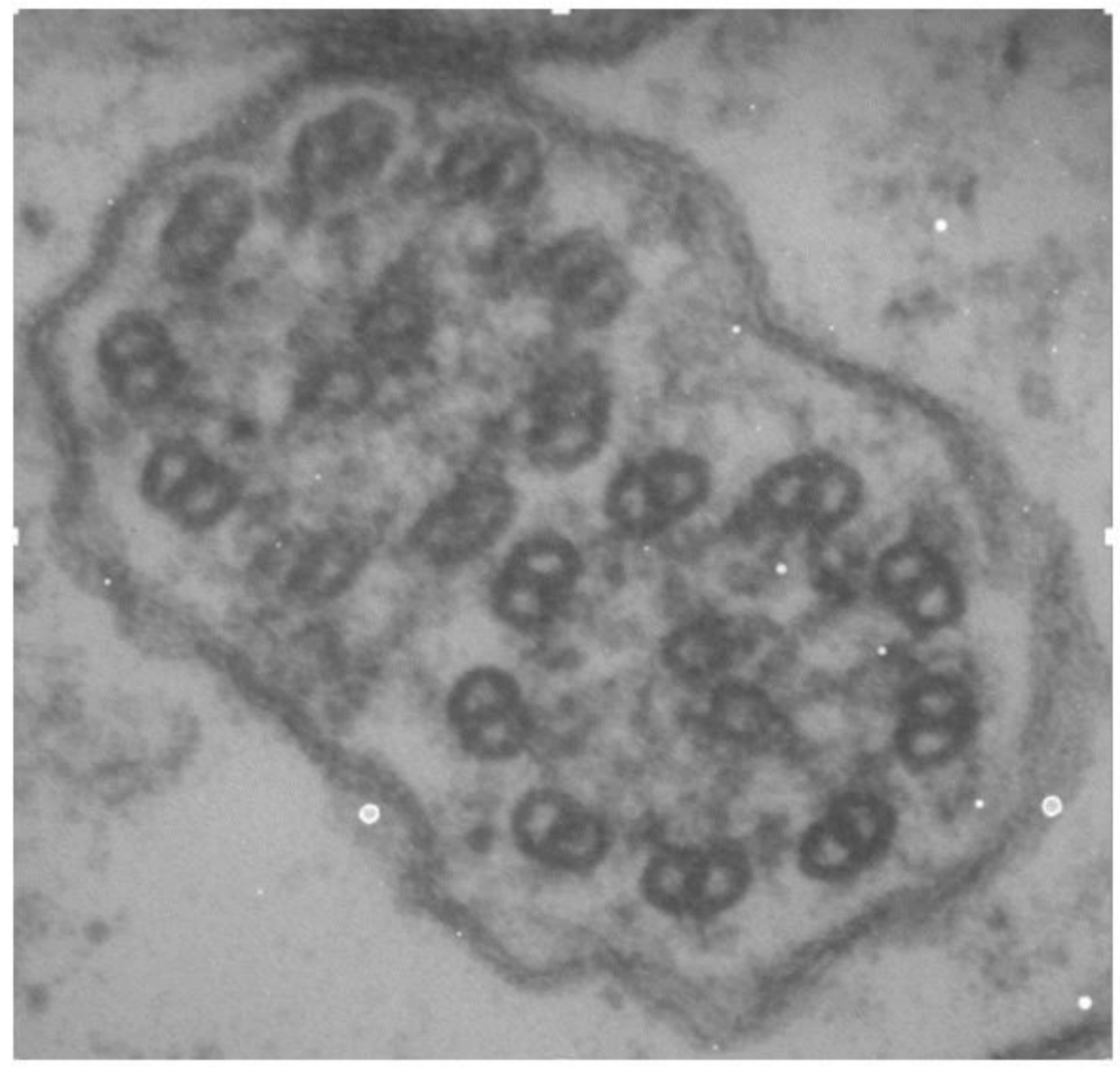

Figure 2

Compound cilia as a SCD 


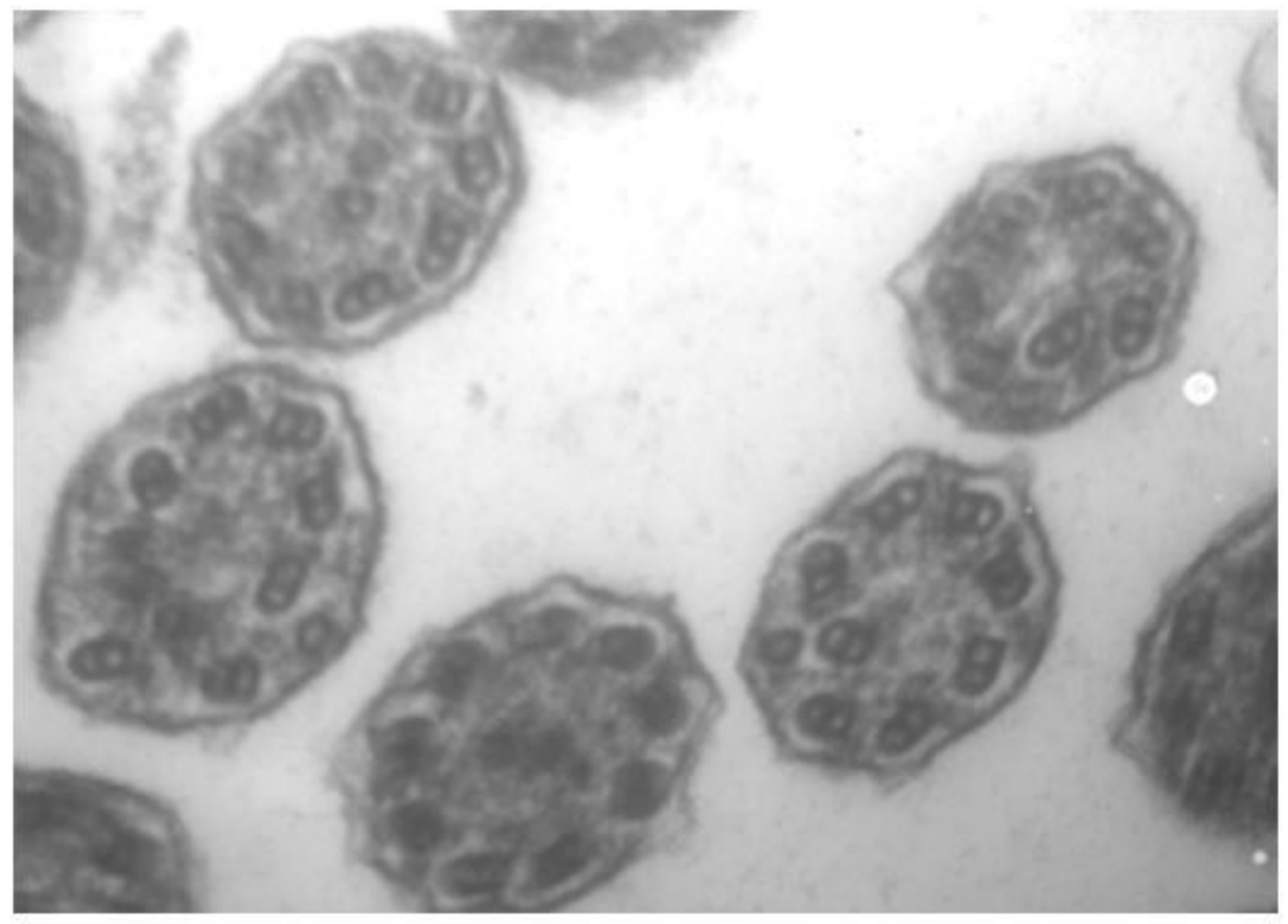

\section{Figure 3}

IDA defect and microtubular disorganization
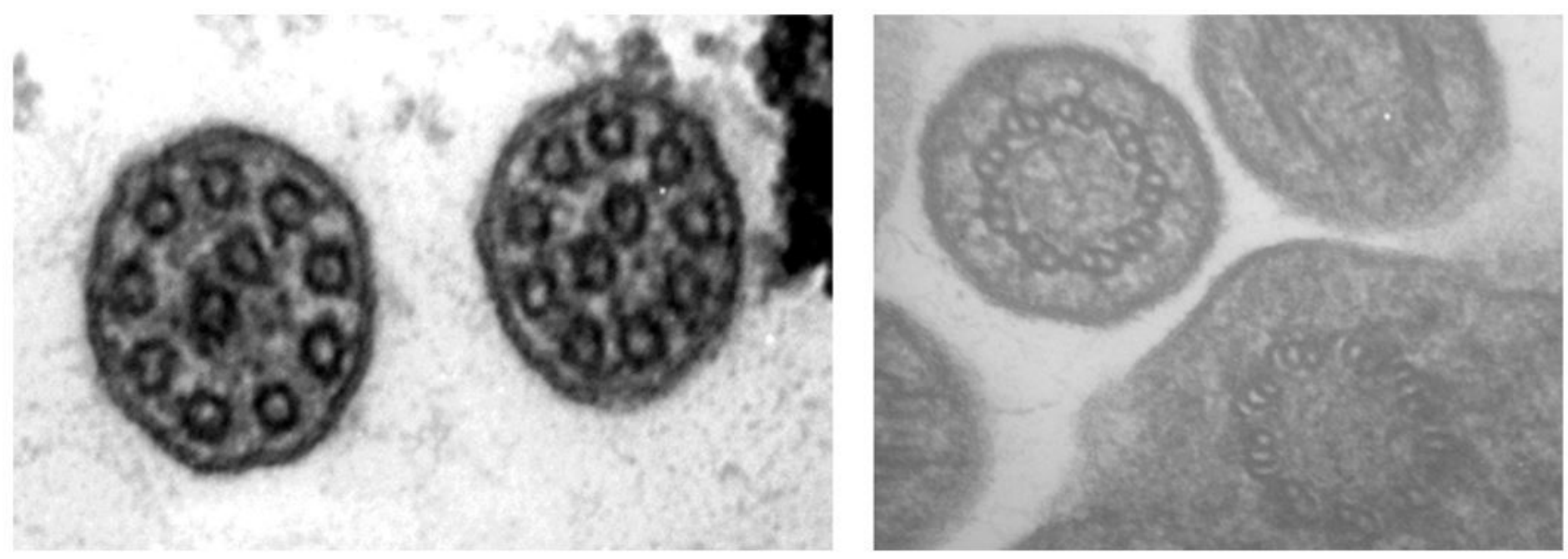

\section{Figure 4}

Sections from the tip (Left) and base (Right) of cilia which shouldn't be misdiagnosed as a defect 


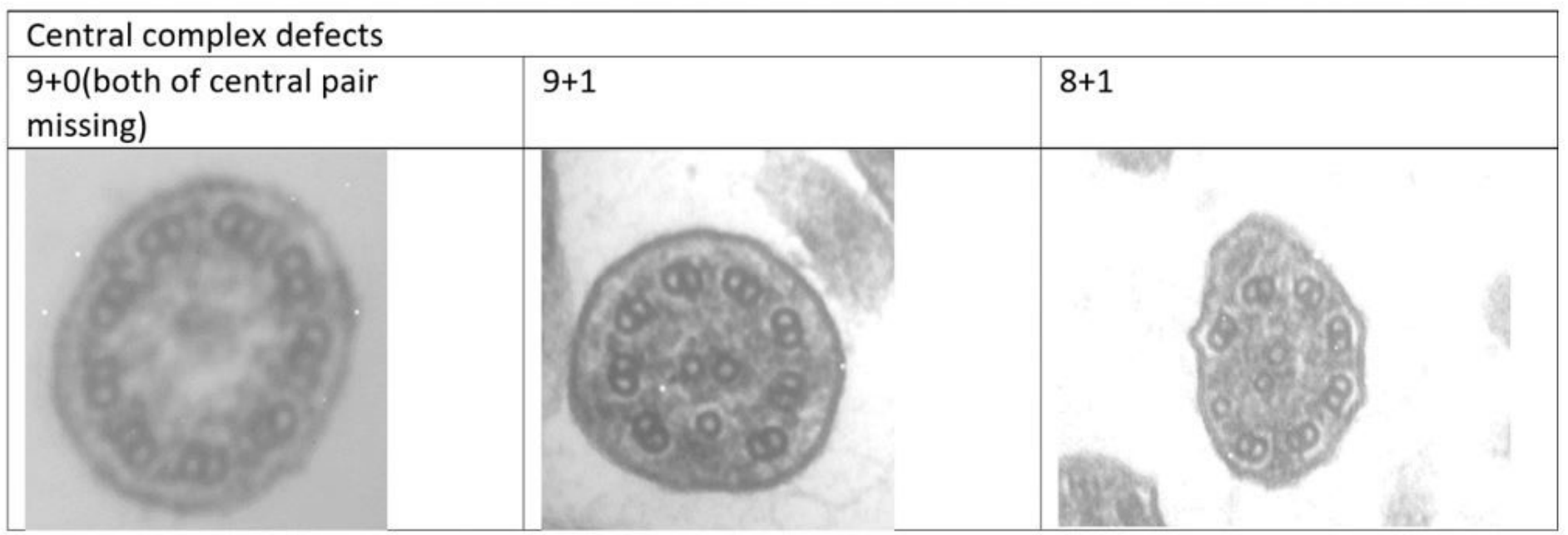

\section{Figure 5}

Variety of central complex defects

\section{Supplementary Files}

This is a list of supplementary files associated with this preprint. Click to download.

- Table3.jpg 